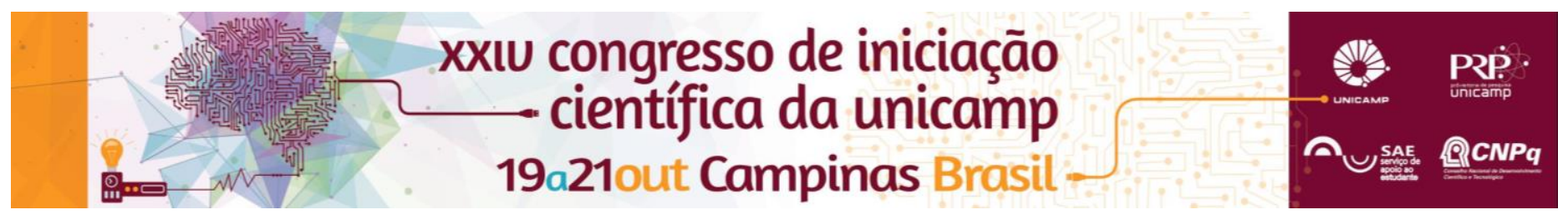

\title{
Chromosomal analysis of two anurans species of the genus Pithecopus (Hylidae, Phyllomedusinae)
}

\author{
Jessika Mayara Lisboa*, Daniel Pacheco Bruschi, Shirlei Maria Recco-Pimentel
}

\begin{abstract}
The genus Pithecopus of the family Hylidae, distributed in the Neotropical region, offers a number of taxonomic and evolutionary possibilities for research. Some of the intrageneric relationships of the representatives of the genus Pithecopus (that includes the species that previously formed the Phyllomedusa hypochondrialis group)are still uncertain. In the present study, the karyotypes of $P$. ayeaye and $P$. palliatus were analyzed for the identification of chromosomal characteristics shared with other species of the genus, and the investigation of features of the chromosomal evolution of the group. The cytogenetic characterization of these species add new information to build hypotheses about the chromosome evolution of the genus Pithecopus.
\end{abstract}

\section{Key words:}

chromosome markers; heterochromatin; NOR

\section{Introduction}

The comparative cytogenetics of anurans provides an important analytical tool for the evaluation of evolutionary and taxonomic questions. Some of the intrageneric relationships of the representatives of the genus Pithecopus (that includes the species that previously belonged to the Phyllomedusa hypochondrialis group), which currently contains 10 species, are still uncertain. In the present study, the karyotypes of $P$. ayeaye and $P$. palliatus were analyzed for the identification of chromosomal characteristics shared with other species of the genus, and the investigation of features of the chromosomal evolution of the group.

\section{Results and Discussion}

Three specimens of $P$. ayeaye and 15 of $P$. palliatus were analyzed. The metaphases were obtained from cellular suspensions of the intestinal epithelium and testicles. The metaphase chromosomes were stained with $10 \%$ Giemsa for the identification of the karyotypes and C-banded to determine the distribution of heterochromatin and silver stained for the identification of the nucleolus organizer regions (Ag-NOR). As in other species of the genus, $P$. palliatus and $P$. ayeaye have a diploid number of $2 n=26$. The karyotypes of both species are composed of four metacentric pairs $(1,4,8$ and 11$)$, six submetacentric pairs (2, 3, 5, 6, 12 and 13) and three subtelocentric pairs (7, 9 and 10). Secondary pericentrometric constrictions were found on the short arms of the homologs of pair 8 in $P$. palliatus, and on the short arms of the homologs of pair 9 in $P$. ayeaye. Secondary interstitial constrictions were also observed on the long arms of only one of the homologs of pairs 3 and 8 in $P$. ayeaye, and in the pericentrometric region of the short arms of one of the homologs of pair 4 . In both species, the secondary constrictions correspond to the NOR sites. In both, $P$. ayeye and $P$. palliatus, the C-banding detected centromeric heterochromatin in all chromosome pairs. Pericentromeric markers were found on the long arms of pairs 6 and 8, and the short arms of pair 11 in $P$. ayeaye, and in pairs 1, 4, 7 and 9 in $P$. palliatus.

\section{Conclusions}

The genus Pithecopus appears to provide an interesting model for the evaluation of the evolutionary dynamics of the NOR sites in the karyotype, given that considerable variability was found in the number and distribution of these features in other species of the genus. Despite the lack of data on the cytogenetics for this group, the chromosomal markers analyzed in the present study provided important insights for the understanding of chromosomal evolution of the genus.

\section{Acknowledgement}

We thank FAPESP for financial support. 